\title{
AN ALGORITHM FOR THE SINGLE-INPUT POLE ASSIGNMENT PROBLEM*
}

\author{
RAFAEL BRU ${ }^{\dagger}$, JOSÉ MAS ${ }^{\dagger}$, AND ANA M. URBANO ${ }^{\dagger}$
}

\begin{abstract}
B. N. Datta and K. Datta have proposed an efficient parallel algorithm for the single-input pole assignment problem when the spectrum set to be assigned is pairwise distinct and disjoint from the spectrum of the dynamical matrix. This paper first presents a theoretical analysis of that algorithm and then presents the necessary modifications on it for an arbitrary spectrum. Based on the modified algorithm, the authors present two new algorithms for the problem and give some results on controllability that are of independent interests.
\end{abstract}

Key words. eigenvalue assignment, single-input, controllability, Jordan form

AMS subject classifications. 93B55, 93B60, 15A18

1. Introduction. Given the time-invariant linear dynamical system

$$
\dot{x}(t)=A x(t)+b u(t),
$$

where $A \in \mathbb{R}^{n \times n}$ and $b \in \mathbb{R}^{n \times 1}$, an important problem in control theory is the eigenvalue assignment problem commonly known as the pole-assignment problem; that is, to find a vector $f$ such that the spectrum of the matrix, $A-b f^{T}$ is equal to the prespecified and conjugated complex number set $\Omega=\left\{\mu_{1}, \mu_{2}, \ldots, \mu_{n}\right\}$. One application of this problem is the stabilization of a controllable system; that is, if the dynamic system has some disturbance, how to choose a vector $f$ such that the closed-loop system comes back to the stable position. It is well known that this problem has a unique solution if and only if the pair $(A, b)$ is controllable [10].

There exist several sequential methods for computing the vector $f$. Some well-known and important methods are found in [1], [2], [5]-[7], and [9].

In [4], B. N. Datta and K. Datta proposed a parallel algorithm for solving the above problem. This algorithm implicitly assumes that the eigenvalues to be assigned are pairwise distinct and different from those of $A$. The main aim of this paper is to modify that method for any self-conjugated set $\Omega$, proposing some modifications of that parallel algorithm to obtain the vector $f$ such that $A-b f^{T}$ has the desired spectrum for any set $\Omega$. We point out here that a multi-input version of the Datta-Datta single-input parallel algorithm was also proposed in [3]. This multi-input version also implicitly assumes that the set of eigenvalues to be assigned is disjoint from the spectrum of $A$ and pairwise distinct.

The paper is organized as follows. First, in $§ 2$ we present a short description of the algorithm given in [4] that is the starting point of our analysis. Then in $\S 3$ we give some controllability results and theoretical analysis of that algorithm proving necessary and sufficient conditions on the validity of that algorithm. In $\S 4$ we study the modification when $\Omega \cap \sigma(A) \neq \varnothing$ (where $\sigma(A)$ denotes the spectrum of $A$ ), and in $\S 5$ we give a general algorithm when $\Omega \cap \sigma(A) \neq \varnothing$ and some eigenvalue has multiplicity greater than one. Finally, in $\$ 6$ we illustrate our algorithms with some numerical experiments with the help of MATLAB.

\footnotetext{
* Received by the editors January 17, 1989; accepted for publication (in revised form) September 21, 1992. This work was supported by Spanish CICYT grant TIC:87-0655.

${ }^{\dagger}$ Departament de Matemàtica Aplicada, Universitat Politècnica de València, Apt. de Correus 22012, 46071, València, Spain (rbru@mat.upv.es).
} 
2. A single-input parallel algorithm for eigenvalue assignment by Datta and Datta. Given the controllable pair $(A, b)$ and the set $\Omega=\left\{\mu_{1}, \mu_{2}, \ldots, \mu_{n}\right\}$, the algorithm proposed in [4] computes a vector $f$ such that $\sigma\left(A-b f^{T}\right)=\Omega$. The algorithm is suitable for parallel implementation and is described as follows.

Stage I. Transform the pair $(A, b)$ by orthogonal similarity to the pair $(H, c)$, where $Q A Q^{T}=H$ is an unreduced upper Hessenberg matrix and $Q b=c=[\alpha, 0, \ldots, 0]^{T}$, $\alpha \neq 0$.

Stage II. Solve the $n$ Hessenberg systems in PARALLEL:

$$
\left(H-\mu_{i} I\right) t_{i}=c, \quad i=1, \quad 2, \ldots, n .
$$

Stage III. Solve for $d$ :

$$
T^{T} d=r
$$

where $T=\left[t_{1}, t_{2}, \ldots, t_{n}\right]$ and $r=[\alpha, \alpha, \ldots, \alpha]^{T}$.

Stage IV. Compute $f^{T}=\alpha^{-1} d^{T} Q$.

In Stage I of the algorithm, the orthogonal similarity $Q$ transforms the pair $(A, b)$ to the equivalent pair $(H, c)$, where $H$ is an upper Hessenberg matrix and $c=[\alpha, 0, \ldots, 0]^{T}$. It is well known that $(A, b)$ is controllable if and only if the matrix $H$ is unreduced (that is, $h_{i, i-1} \neq 0, i=2, \ldots, n$ ) and $\alpha \neq 0$ (see [6] and [7]).

Since

$$
Q\left(A-b f^{T}\right) Q^{T}=H-c f^{T} Q^{T}
$$

the problem of finding the vector $f$ such that $\sigma\left(A-b f^{T}\right)=\Omega$ is equivalent to finding the vector $g$, defined by $g^{T}=f^{T} Q^{T}$, such that

$$
\sigma\left(H-c g^{T}\right)=\Omega .
$$

Stage II of the algorithm solves the systems

$$
\left(H-\mu_{i} I\right) t_{i}=c, \quad i=1, \quad 2, \ldots, n .
$$

Assuming that $T$ is nonsingular, it computes the vector $d^{T}=r^{T} T^{-1}$ in Stage III.

In Stage IV the vector $f$ is computed as

$$
f^{T}=\alpha^{-1} d^{T} Q=[1,1, \ldots, 1] T^{-1} Q=g^{T} Q .
$$

Theorem 2.2 of [4] proves that the above algorithm provides the vector $f$ such that $\sigma\left(A-b f^{T}\right)=\Omega$, assuming that the matrix $T$ obtained in Stage II is invertible. We prove that the nonsingularity of $T$ is guaranteed when the elements of the set $\Omega$ are pairwise distinct and when no element of $\Omega$ is in $\sigma(A)$ (see Proposition 3). Thus the above assumptions in the algorithm given in [4] are not a restriction because it works implicitly with these conditions. As we shall see in the next section, the nonsingularity of $T$ is related with the consistency of the systems $(H-\mu I) t=c$ of Stage II.

3. Consistency and nonsingularity. In general, the matrix $T$ obtained by solving (1) is not invertible if some eigenvalue has multiplicity greater than one as Proposition 1 shows. Furthermore, in the case that $\Omega \cap \sigma(A) \neq \varnothing$, the matrix $T$ cannot be constructed from the systems of Stage II, as can be seen in Proposition 2. From the above remarks, we observe that the algorithm given in [4] is valid if and only if $\Omega \cap \sigma(A)=\varnothing$ and $\Omega$ is a pairwise disjoint set, as the following theorem proves. Since $\sigma(A)=\sigma(H)$, we state our results for an unreduced upper Hessenberg matrix $H$. 
THEOREM 1. Let $(H, c)$ be a controllable pair. Let $\Omega$ be a conjugated complex number set. The algorithm given in [4] is valid if and only if the following conditions are satisfied: (i) $\Omega \cap \sigma(H)=\varnothing$, and (ii) the elements of $\Omega$ are pairwise distinct.

The proof of Theorem 1 is based on the following results.

Proposition 1. Let $(H, c)$ be a controllable pair. Let $\Omega=\left\{\mu_{1}, \mu_{2}, \ldots, \mu_{n}\right\}$ be a conjugated complex number set. If $\Omega \cap \sigma(H)=\varnothing$, but $\mu_{i}=\mu_{j}$ for some $i \neq j$, then the matrix T constructed in Stage II is singular.

Proof. Since $\Omega \cap \sigma(H)=\varnothing$, then rank $\left(H-\mu_{i} I\right)=n$. Therefore, the system $\left(H-\mu_{i} I\right) x=c$ has a unique solution and hence the matrix $T$ has the $i$ th and $j$ th columns equal. Hence $T$ is singular.

The following lemma gives a basic result on the controllable systems with single input, and we will use it in the next proposition. Moreover, the remark below is the main point for constructing the general algorithm given in $\S 5$.

LEMMA 1. Let $(H, c)$ be a controllable pair. Then

(i) $\operatorname{dim} \operatorname{Ker}[H-\mu I, c]=1$, for all $\mu$.

(ii) If $\mu \in \sigma(H)$, then the geometric multiplicity of $\mu$ is 1 .

Proof. (i) If $\mu \notin \sigma(H)$, it is obvious. Otherwise, since $(H, c)$ is controllable, rank $[H-\mu I, c]=n$ and hence $\operatorname{dim} \operatorname{Ker}[H-\mu I, c]=1$.

(ii) Since rank $[H-\mu I, c]=n$ and considering that $\mu \in \sigma(H)$, we deduce that dim $\operatorname{Ker}(H-\mu I)=1$.

Remark. Let $\gamma_{j}$ be the $j$ th column of the matrix $H-\mu I, j=1,2, \ldots, n$.

(a) Then by Lemma 1(ii), and since $H-\mu I$ is an unreduced upper Hessenberg matrix, we have

$$
\operatorname{span}\left\{\gamma_{1}, \gamma_{2}, \ldots, \gamma_{n-1}, \gamma_{n}\right\}=\operatorname{span}\left\{\gamma_{1}, \gamma_{2}, \ldots, \gamma_{n-1}\right\} \text {. }
$$

(b) By Lemma 1(i) and the last remark, we have the following direct sums:

$$
\begin{aligned}
\mathbb{C}^{n} & =\operatorname{span}\left\{\gamma_{1}, \gamma_{2}, \ldots, \gamma_{n-1}, \gamma_{n}\right\} \oplus \operatorname{span}\{c\} \\
& =\operatorname{span}\left\{\gamma_{1}, \gamma_{2}, \ldots, \gamma_{n-1}\right\} \oplus \operatorname{span}\{c\} .
\end{aligned}
$$

Proposition 2. Consider the controllable pair $(H, c)$. Let $\Omega$ be a conjugated complex number set. If $\mu \in \Omega \cap \sigma(H)$, then the system $(H-\mu I) x=c$ is inconsistent.

Proof. If $\mu \in \sigma(H)$, then by Lemma 1(ii), we have that rank $(H-\mu I)=n-1$, and since $(H, c)$ is a controllable pair, rank $[H-\mu I, c]=n$. Thus, the system $(H-\mu I) x=c$ is inconsistent.

For the next proposition we consider, for each $p=1,2, \ldots, n$, the following partition of the matrix $H-\mu_{i} I$ and the vectors $t_{i}$ and $c$.

$$
\begin{gathered}
H-\mu_{i} I=\left[\frac{H_{1}-\mu_{i} I}{R} \mid \frac{S}{H_{2-p}-\mu_{i} I}\right]_{p}^{n-p} \\
t_{i}=\left[\begin{array}{l}
t_{1 i} \\
\cdot \\
\cdot \\
\frac{t_{n-p, i}}{t_{n-p+1, i}} \\
\cdot \\
t_{n, i}
\end{array}\right]=\left[\frac{t_{i}^{n-p}}{t_{i}^{p}}\right], \quad c=\left[\begin{array}{c}
\alpha \\
0 \\
\cdot \\
\cdot \\
\frac{0}{0} \\
\cdot \\
0
\end{array}\right]=\left[\frac{c^{n-p}}{0}\right]_{p}^{n-p}
\end{gathered}
$$

for each $i=1,2, \ldots, p$. 
Proposition 3. Let $(H, c)$ be a controllable pair. Let $\Omega=\left\{\mu_{1}, \mu_{2}, \ldots, \mu_{n}\right\}$ be a conjugated complex number set, pairwise distinct. Consider, in addition, that $\Omega \cap$ $\sigma(H)=\varnothing$. Let $t_{i}$ be the solution of the system

$$
\left(H-\mu_{i} I\right) t_{i}=c \text { for each } i=1,2, \ldots, n .
$$

Then the rank of the matrix $\left[t_{1}^{p}, t_{2}^{p}, \ldots, t_{p}^{p}\right]$ is equal to $p$ for each $p=1,2, \ldots, n$.

Proof. We proceed by induction over $p$. For $p=1$, it is evident that $t_{1 n} \neq 0$. Otherwise, since $H$ is unreduced Hessenberg matrix and using back substitution we deduce that the vector $t_{1}$ is the zero vector, which is not a solution of the nonhomogeneous system $\left(H-\mu_{1} I\right) x=c$.

Suppose by induction that the rank of the matrix $\left[t_{1}^{\nu}, t_{2}^{\nu}, \ldots, t_{p}^{p}\right]$ is $p$. Obviously, the rank of the matrix

$$
\left[t_{1}^{p+1}, t_{2}^{p+1}, \ldots, t_{p}^{p+1}, t_{p+1}^{p+1}\right]
$$

is $p$ or $p+1$. Suppose the rank of this matrix is $p$ instead of $p+1$. Then by the induction hypothesis we have the following linear combination:

$$
t_{p+1}^{p+1}=\sum_{j=1}^{p} \beta_{j} t_{j}^{p+1}
$$

For each $i=1,2, \ldots, p+1$, by the above partitions, we can write system (2) as

$$
\left[\frac{H_{1}-\mu_{i} I}{R} \mid \frac{S}{H_{2}-\mu_{i} I}\right]\left[\frac{t_{i}^{n-p-1}}{t_{i}^{p+1}}\right]=\left[\frac{c^{n-p-1}}{0}\right] .
$$

Now restricting ourselves to the second subsystem of (4), for $i=1,2, \ldots, p$, we have

$$
R t_{i}^{n-p-1}+\left(H_{2}-\mu_{i} I\right) t_{i}^{p+1}=0,
$$

and for $i=p+1$, taking into account (3), we get

$$
R t_{p+1}^{n-p-1}+\left(H_{2}-\mu_{p+1} I\right) \sum_{j=1}^{p} \beta_{j} t_{j}^{p+1}=0 .
$$

Subtracting expression (6) from the sum of all $p$ subsystems (5) each one multiplied by $\beta_{i}, i=1,2, \ldots, p$, we obtain

$$
R\left[\sum_{i=1}^{p} \beta_{i} t_{i}^{n-p-1}-t_{p+1}^{n-p-1}\right]+\sum_{i=1}^{p} \beta_{i}\left(\mu_{p+1}-\mu_{i}\right) t_{i}^{p+1}=0 .
$$

Since the matrix $R$ has only the $(1, n-p)$ th entry different from zero, we have that the last $p$ rows satisfy

$$
\sum_{i=1}^{p} \beta_{i}\left(\mu_{p+1}-\mu_{i}\right) t_{i}^{p}=0
$$

Hence the rank of matrix $\left[t_{1}^{p}, t_{2}^{p}, \ldots, t_{p}^{p}\right]$ is less than $p$, which contradicts the induction hypothesis.

We are now ready to prove Theorem 1.

Proof of Theorem 1. Sufficiency: Follows from Proposition 3. Necessity: If $\Omega \cap$ $\sigma(H) \neq \varnothing$, by Proposition 2, the matrix $T$ cannot exist. On the other hand if multiplicity of $\mu>1$, for some $\mu \in \Omega$, by Proposition $1, \mathrm{~T}$ is singular. 
4. Proposed pole placement algorithm. If $\mu \in \Omega \cap \sigma(H)$, we then have seen in Proposition 2 that the Datta-Datta algorithm is not valid. However, it is always possible to find an eigenvector of $H$ associated with $\mu$, solving the system $(H-\mu I) x=0$. In fact, we shall see in Theorem 2 that in this case (that is, when $\Omega \cap \sigma(H) \neq \varnothing$ ) the matrix $T$ constructed in (7) is nonsingular. We will thus have the following modified algorithm.

\section{ALGORITHM I}

Stage I. Transform the pair $(A, b)$ by orthogonal similarity to the pair $(H, c)$, where $H$ is an upper unreduced Hessenberg matrix and $c=[\alpha, 0, \ldots, 0]^{T}$, with $\alpha \neq 0$.

Stage II. For $i=1,2, \ldots, n$.

If $\mu_{i} \notin \sigma(H)$, then

Solve the Hessenberg systems in PARALLEL

$$
\left(H-\mu_{i} I\right) t_{i}=c .
$$

Else

Solve the Hessenberg systems in PARALLEL for the nonzero solution

$$
\left(H-\mu_{i} I\right) t_{i}=0 .
$$

Stage III. Form the vector $u$ as follows: assign 1 to the $i$ th entry if $\mu_{i} \in \sigma(H)$, otherwise assign 0 to this entry.

Stage IV. Solve for $g$ :

$$
g^{T} T=u^{T}
$$

where

$$
T=\left[t_{1}, t_{2}, \ldots, t_{n}\right]
$$

Stage V. Compute $f^{T}=g^{T} Q$.

The following result proves the validity of the above algorithm.

THEOREM 2. Let $(H, c)$ be a controllable pair and let $\Omega=\left\{\mu_{1}, \mu_{2}, \ldots, \mu_{n}\right\}$ be a conjugated complex number set pairwise distinct. If $t_{i}, i=1,2, \ldots, n, i$ s a solution of the systems

$$
\left(H-\mu_{i} I\right) t_{i}= \begin{cases}0 & \text { if } \mu_{i} \in \sigma(H), \\ c & \text { if } \mu_{i} \notin \sigma(H),\end{cases}
$$

where $c=[\alpha, 0, \ldots, 0]^{T}, \alpha \neq 0$, then the matrix

$$
\left[t_{1}^{p}, t_{2}^{p}, \ldots, t_{p}^{p}\right]
$$

has rank $p$, for each $p=1,2, \ldots, n$.

Proof. The proof is analogous to that of Proposition 3.

The assumption that the complex numbers of $\Omega$ are pairwise distinct in Theorem 2 is necessary as the following example shows.

Example 1. Let

$$
H=\left[\begin{array}{lll}
1 & 0 & 0 \\
1 & 1 & 0 \\
0 & 1 & 1
\end{array}\right] \text { and } c=\left[\begin{array}{l}
1 \\
0 \\
0
\end{array}\right]
$$


and suppose that we want to assign the spectrum $\Omega=\{1,1,2\}$. The solution of the systems (7) yields the singular matrix

$$
T=\left[\begin{array}{rrr}
0 & 0 & -1 \\
0 & 0 & -1 \\
1 & 1 & 1
\end{array}\right] .
$$

5. The general algorithm. With the hypothesis of Theorem 2 , solving the systems (7) is equivalent to solving the matrix system

$$
H T-c u^{T}=T \operatorname{diag}\left(\mu_{i}\right),
$$

where $u^{T}$ is a row vector constructed in the proposed algorithm: its $i$ th entry is 0 if $\mu_{i} \in$ $\sigma(H)$ and 1 otherwise. From (8) note that the matrix $H-c u^{T} T^{-1}$ is diagonalizable as in the case of [4]. However, in the general case where the spectrum to be assigned has some eigenvalue with multiplicity greater than one, the matrix $H-c u^{T} T^{-1}$ is not similar to a diagonal matrix as the following proposition shows.

Proposition 4. Let $(H, c)$ be a controllable pair, and let $\Omega=\left\{\mu_{1}, \mu_{2}, \ldots, \mu_{p}\right\}$ be the spectrum to be assigned with multiplicities $m(1), m(2), \ldots, m(p)$, respectively. If $g$ is the vector such that $\sigma\left(H-c g^{T}\right)=\Omega$, then the Jordan form of $H-c g^{T}$ is

$$
J=\left[\begin{array}{llll}
J_{1} & 0 & \cdots & 0 \\
0 & J_{2} & \cdots & 0 \\
\cdots & \cdots & \cdots & \cdots \\
0 & 0 & \cdots & J_{p}
\end{array}\right],
$$

where $J_{k}$ is the $m(k) \times m(k)$ Jordan matrix

$$
J_{k}=\left[\begin{array}{ccccc}
\mu_{k} & 0 & \cdots & 0 & 0 \\
1 & \mu_{k} & \cdots & 0 & 0 \\
\cdots & \cdots & \cdots & \cdots & \cdots \\
0 & 0 & \cdots & 1 & \mu_{k}
\end{array}\right] .
$$

Proof. Let $\mu \in \sigma\left(H-c g^{T}\right)$; we need to prove that $\operatorname{dim} \operatorname{Ker}\left(H-c g^{T}-\mu I\right)=1$. Suppose that $t_{1}$ and $t_{2}$ are solutions of $\left(H-c g^{T}-\mu I\right) x=0$. Then

$$
(H-\mu I) t_{i}=c g^{T} t_{i}=\xi_{i} c, \quad i=1,2 .
$$

Now we distinguish two cases. (i) $\mu \in \sigma(H)$. Then $\xi_{i}=0, i=1,2$, otherwise the systems (10) are inconsistent by Proposition 2. Therefore the vectors $t_{1}$ and $t_{2}$ are in $\operatorname{Ker}(H-\mu I)$ which, by Lemma 1(ii), has dimension 1 . Hence the vectors $t_{1}$ and $t_{2}$ are linearly dependent.

(ii) $\mu \notin \sigma(H)$. In this case $\xi_{i} \neq 0, i=1,2$ (otherwise the unique solution is the trivial one). Equation (10) becomes

$$
(H-\mu I)\left(\xi_{i}^{-1} t_{i}\right)=c, \quad i=1,2 .
$$

Since the system $(H-\mu I) x=c$ has unique solution, then $t_{1}$ and $t_{2}$ are linearly dependent. We conclude that $\operatorname{dim} \operatorname{Ker}\left(H-c g^{T}-\mu I\right)=1$.

Remark. Proposition 4 suggests that when there are in the set $\Omega$ eigenvalues with multiplicity greater than one, the matrix diag $\left(\mu_{i}\right)$ in $(8)$ may be substituted by the matrix $J$ defined in (9) to compute the matrix $T$. Now we discuss how to choose the vector $u$ so that the matrix system

$$
H T-c u^{T}=T J
$$

provides $n$ consistent linear systems. Later, we shall see in Theorem 3 that the matrix $\mathrm{T}$ constructed from the solutions of these consistent systems is invertible. 
Remark. To see the consistency of the system (11) as a function of the vector $u$, we partition this vector as

$$
u=\left[u_{(1)}^{T}, u_{(2)}^{T}, \ldots, u_{(p)}^{T}\right]^{T}
$$

where the $i$ th block of $u^{T}$ is

$$
u_{(i)}=\left[\beta_{1}^{(i)}, \beta_{2}^{(i)}, \ldots, \beta_{m(i)}^{(i)}\right]^{T}
$$

and $m(i)$ is the multiplicity of $\mu_{i}$. Also we write the matrix $T$ by blocks as

$$
T=\left[t_{(1)}, t_{(2)}, \ldots, t_{(p)}\right]
$$

where the block $t_{(i)}$ is

$$
t_{(i)}=\left[t_{1}^{(i)}, t_{2}^{(i)}, \ldots, t_{m(i)}^{(i)}\right]
$$

for $i=1,2, \ldots, p$. The superscripts represent the corresponding eigenvalues. From the matrix system (11), using this notation, we obtain the following linear systems:

$$
\begin{gathered}
\left(H-\mu_{i} I\right) t_{j}^{(i)}=t_{j+1}^{(i)}+\beta_{j}^{(i)} c, \\
j=1,2, \ldots, m(i), \quad i=1,2, \ldots, p,
\end{gathered}
$$

where $t_{m(i)+1}^{(i)}=0$.

The systems (12) can be grouped into $p$ subsystems corresponding to each eigenvalue $\mu_{i}, i=1,2, \ldots, p$. Let us study the consistency of a subsystem (the same study can be done for the other subsystems). To simplify the notation, we drop the scripts ( $i$ ) and work with the linear systems

$$
(H-\mu I) t_{j}=t_{j+1}+\beta_{j} c, \quad j=m, m-1, \ldots, 1,
$$

where $t_{m+1}=0$.

We consider two cases: (i) $\mu \notin \sigma(H)$. In this case $H-\mu I$ is nonsingular and the $m$ linear systems (13) have unique solutions. The last system (i.e., $j=m$ )

$$
(H-\mu I) t_{m}=\beta_{m} c
$$

is consistent for every scalar $\beta_{m}$. In particular, we obtain a nontrivial solution for $\beta_{m}=$ 1. The remaining systems are consistent for any value of $\beta_{j}$; in particular, we shall take $\beta_{j}=0, j=m-1, m-2, \ldots, 2,1$.

(ii) $\mu \in \sigma(H)$. Suppose that the multiplicity in $\sigma(H)$ is $r$. Since $(H, c)$ is controllable, by Lemma 1(ii) the $r$ systems

$$
\begin{gathered}
(H-\mu I) t_{m}=0, \\
(H-\mu I) t_{m-1}=t_{m}, \\
\cdots \ldots \ldots \cdots \cdots \\
(H-\mu I) t_{m-r+1}=t_{m-r+2}
\end{gathered}
$$

are consistent.

(iia) In the case $m \leq r$, then the systems (13) are consistent for $\beta_{m}=\beta_{m-1}=$ $\cdots=\beta_{1}=0$. 
Exploiting the structure of the unreduced Hessenberg matrix, a particular solution of the systems (13) has the structure

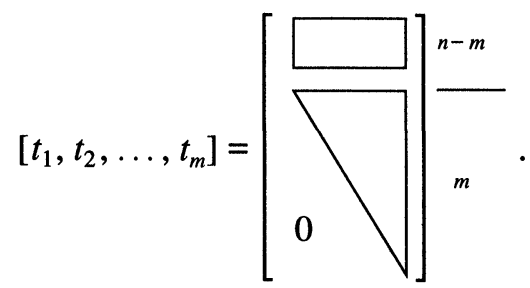

(iib) In the case $m>r$, the first $r$ systems (13) are consistent for $\beta_{m}=\beta_{m-1}=$ $\cdots=\beta_{m-r+1}=0$. Then the next system of (13) to solve is

$$
(H-\mu I) t_{m-r}=t_{m-r+1}+\beta_{m-r} c,
$$

or equivalently

$$
(H-\mu I) t_{m-r}-\beta_{m-r} c=t_{m-r+1},
$$

which is equivalent to

$$
[H-\mu I, c]\left[\begin{array}{l}
\delta_{1} \\
\delta_{2} \\
\vdots \\
\delta_{n} \\
\Gamma_{m-r}
\end{array}\right]=t_{m-r+1}
$$

By the remark of Lemma 1, we can also write

$$
t_{m-r+1}=\delta_{1} \gamma_{1}+\delta_{2} \gamma_{2}+\cdots+\delta_{n-1} \gamma_{n-1}+\Gamma_{m-r} c
$$

where $\gamma_{i}$ represents the $i$ th column of the matrix $H-\mu I, i=1,2, \ldots, n-1$. Hence we can find a particular solution of (17) setting $\delta_{n}=0$. Then a solution of (16) is $\beta_{m-r}=-\Gamma_{m-r}$ and

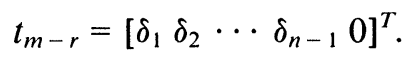

The other vectors $t_{m-r-1}, t_{m-r-2}, \ldots, t_{1}$ can similarly be obtained.

Again, by the structure of the matrix $[H-\mu I, c]$, we can get a partial solution of (13) with the following structure:

$$
\left[t_{1}, \ldots, t_{r}, t_{r+1}, \ldots, t_{m}\right]=\left[\begin{array}{l}
\square \\
0
\end{array}\right]^{n-m} .
$$

Example 2. Let

$$
H=\left[\begin{array}{rrr}
2 & 0 & 0 \\
1 & 1 & 0 \\
0 & 1 & -1
\end{array}\right] \text { and } c=\left[\begin{array}{l}
1 \\
0 \\
0
\end{array}\right]
$$


Suppose that we want to assign the spectrum $\Omega=\{1,1,1\}$. Since $1 \in \sigma(H)$, with multiplicity 1 , we are in the case (iib), and we must solve the systems

$$
\begin{gathered}
(H-I) t_{3}=0, \\
(H-I) t_{2}=t_{3}+\beta_{2} c, \\
(H-I) t_{1}=t_{2}+\beta_{1} c .
\end{gathered}
$$

A solution of the first system is $t_{3}=[0,2,1]^{T}$. By (16) we get $\beta_{2}=2$, so a solution of the second system will be $t_{2}=[2,1,0]^{T}$. With this solution and (17), we obtain $\beta_{1}=$ -1 and then one solution of the last system will be $t_{1}=[1,0,0]^{T}$, and hence $u=$ $[-1,2,0]^{T}$.

\section{AlgorithM II}

This algorithm finds the vector $f$ such that given the controllable pair $(A, b)$ and the conjugated complex number set $\Omega=\left\{\mu_{1}, \mu_{2}, \ldots, \mu_{p}\right\}$, the matrix $A-b f^{T}$ has the spectrum $\Omega$, where $\mu_{i}$ has multiplicity $m(i), i=1,2, \ldots, p$. For the description of the algorithm it is assumed that the spectrum of $A$ (and so the spectrum of $H$ ) and the multiplicities $r(1), r(2), \ldots, r(m)$ of its eigenvalues are known.

In the algorithm we represent by $u_{(s)}$ the block of the vector $u$ that has coordinates from $m(1)+\cdots+m(s-1)+1$ to $m(1)+\cdots+m(s-1)+m(s)$, and by $\gamma_{j}^{(i)}, j=1$, $2, \ldots, n$ the columns of matrix $H-\mu_{i} I$.

Stage I. Transform the pair $(A, b)$ by orthogonal similarity $Q$ to the pair $(H, c)$, where $H$ is an upper unreduced Hessenberg matrix and $c=[\alpha, 0, \ldots, 0]^{T}$, with $\alpha \neq 0$.

Stage II. For $i=1,2, \ldots, p$.

If $\mu_{i} \notin \sigma(H)$.

Solve the system

$$
\left(H-\mu_{i} I\right) t_{m(i)}^{(i)}=c .
$$

For $j=m(i)-1, m(i)-2, \ldots, 1$ solve the systems

$$
\left(H-\mu_{i} I\right) t_{j}^{(i)}=t_{j+1}^{(i)} .
$$

Form the $m(i)$-dimensional vector $u_{(i)}=[0,0, \ldots, 0,1]^{T}$. Else

If $r(i) \geq m(i)$, solve the system for the nonzero solution

$$
\left(H-\mu_{i} I\right) t_{m(i)}^{(i)}=0 .
$$

For $j=m(i)-1, m(i)-2, \ldots, 1$ solve the systems

$$
\left(H-\mu_{i} I\right) t_{j}^{(i)}=t_{j+1}^{(i)} \text {. }
$$

Form the $m(i)$-dimensional vector $u_{(i)}=[0,0, \ldots, 0]^{T}$.

Else solve the system for the nontrivial solution

$$
\left(H-\mu_{i} I\right) t_{m(i)}^{(i)}=0 .
$$

For $j=m(i)-1, m(i)-2, \ldots, m(i)-r(i)+1$ solve the systems

$$
\left(H-\mu_{i} I\right) t_{j}^{(i)}=t_{j+1}^{(i)} \text {. }
$$

For $j=m(i)-r(i), m(i)-r(i)-1, \ldots, 2,1$, solve the systems

$$
t_{j+1}^{(i)}=\delta_{1}^{(i)} \gamma_{1}^{(i)}+\delta_{2}^{(i)} \gamma_{2}^{(i)}+\cdots+\delta_{n-1}^{(i)} \gamma_{n-1}^{(i)}+\Gamma_{j}^{(i)} c .
$$


Form the $m(i)$-dimensional vector

$$
u_{(i)}=\left[u_{1}^{(i)}, u_{2}^{(i)}, \ldots, u_{m(i)}^{(i)}\right]
$$

where $u_{j}^{(i)}=-\Gamma_{j}^{(i)}, j=1,2, \ldots, m(i)-r(i)$, and 0 elsewhere.

Form $t_{(j)}=\left[\delta_{1}^{(i)}, \delta_{2}^{(i)}, \ldots, \delta_{n-1}^{(i)}, 0\right]^{T}$.

Stage III. Form the vector $u=\left[u_{(1)}, u_{(2)}, \ldots, u_{(p)}\right]^{T}$.

Stage IV. Solve for $g$ :

$$
g^{T} T=u^{T}
$$

where

$$
T=\left[t_{(1)}, t_{(2)}, \ldots, t_{(p)}\right]
$$

and $t_{(i)}$ is

$$
t_{(i)}=\left[t_{1}^{(i)}, t_{2}^{(i)}, \ldots, t_{m(i)}^{(i)}\right] .
$$

Stage V. Compute $f^{T}=g^{T} Q$.

The following theorem proves the validity of Algorithm II.

THEOREM 3. Let $(H, c)$ be a controllable pair. Let $\Omega=\left\{\mu_{1}, \mu_{2}, \ldots, \mu_{p}\right\}$ be an arbitrary conjugated complex number set. Suppose that the multiplicity of $\mu_{i}$ is $m(i)$ and $r(i)$ in $\Omega$ and $\sigma(H)$, respectively, $i=1,2, \ldots, p$. Then the solutions of the systems

$$
\begin{gathered}
\left(H-\mu_{i} I\right) t_{j}^{(i)}=t_{j+1}^{(i)}+\beta_{j}^{(i)} c, \\
j=1,2, \ldots, m(i), \quad i=1,2, \ldots, p
\end{gathered}
$$

are linearly independent, where

(a) $t_{m(i)+1}^{(i)}=0$.

(b) If $\mu_{i} \notin \sigma(H)$, then $\beta_{m(i)}^{(i)}=1$ and $\beta_{j}^{(i)}=0$ for $j=m(i)-1, m(i)-2, \ldots, 2,1$.

(c) If $\mu_{i} \in \sigma(H)$ and

(c1) $r(i) \geq m(i)$, then $\beta=0, j=m(i), m(i)-1, \ldots, 2,1$.

(c2) $r(i)<m(i)$, then $\beta_{j}^{(i)}=0, j=m(i), \ldots, m(i)-r(i)+1$ and the others $\beta_{j}^{(i)}$ are calculated by (16).

Proof. We need to prove that the matrix

$$
T=\left[t_{(1)}, t_{(2)}, \ldots, t_{(p)}\right]
$$

where

$$
t_{(i)}=\left[t_{1}^{(i)}, t_{2}^{(i)}, \ldots, t_{m(i)}^{(i)}\right], \quad i=1,2, \ldots, p
$$

is nonsingular. We will proceed in three steps.

Step 1. $\mu_{i} \notin \sigma(H)$. We can proceed by induction on $m(i)$ as in the proof of Proposition 3. We start in this case in the reverse order because the first linear system we solve is $\left(H-\mu_{i} I\right) t_{m(i)}^{(i)}=c$. Then we obtain that the rank of

$$
t_{(i)}=\left[t_{1}^{(i)}, t_{2}^{(i)}, \ldots, t_{m(i)}^{(i)}\right]
$$

corresponding to this eigenvalue is $m(i)$.

Step 2. $\mu_{i} \in \sigma(H)$. Taking into account the structure of the particular solutions (14) and (18), the block

$$
t_{(i)}=\left[t_{1}^{(i)}, t_{2}^{(i)}, \ldots, t_{m(i)}^{(i)}\right]
$$

has rank $m(i)$.

Step 3. It remains to prove the independence of the blocks $t_{(i)}$ of $T$ corresponding to the eigenvalues $\mu_{i}$ for all $i=1,2, \ldots, p$. For that, proceeding similarly to the proof 
of Proposition 3, we obtain that $T$ is nonsingular. In this case, again, the induction is applied in the reverse order as Step 1 of this theorem.

6. Results. In this section we present numerical results obtained by our algorithms on two test problems: the Frank and Wilkinson matrices. These matrices were used in [3] as test matrices.

The accuracy obtained compares favorably with that obtained by Datta and Datta [4] in their algorithm. We performed experiments with Algorithms I and II. MATLAB [8] was used for implementing our algorithms.

Example 1. The Wilkinson matrix

$W=\left[\begin{array}{rrrrrrrrrrrrrrrrrrrr}20 & 0 & 0 & 0 & 0 & 0 & 0 & 0 & 0 & 0 & 0 & 0 & 0 & 0 & 0 & 0 & 0 & 0 & 0 & 0 \\ 20 & 19 & 0 & 0 & 0 & 0 & 0 & 0 & 0 & 0 & 0 & 0 & 0 & 0 & 0 & 0 & 0 & 0 & 0 & 0 \\ 0 & 20 & 18 & 0 & 0 & 0 & 0 & 0 & 0 & 0 & 0 & 0 & 0 & 0 & 0 & 0 & 0 & 0 & 0 & 0 \\ 0 & 0 & 20 & 17 & 0 & 0 & 0 & 0 & 0 & 0 & 0 & 0 & 0 & 0 & 0 & 0 & 0 & 0 & 0 & 0 \\ 0 & 0 & 0 & 20 & 16 & 0 & 0 & 0 & 0 & 0 & 0 & 0 & 0 & 0 & 0 & 0 & 0 & 0 & 0 & 0 \\ 0 & 0 & 0 & 0 & 20 & 15 & 0 & 0 & 0 & 0 & 0 & 0 & 0 & 0 & 0 & 0 & 0 & 0 & 0 & 0 \\ 0 & 0 & 0 & 0 & 0 & 20 & 14 & 0 & 0 & 0 & 0 & 0 & 0 & 0 & 0 & 0 & 0 & 0 & 0 & 0 \\ 0 & 0 & 0 & 0 & 0 & 0 & 20 & 13 & 0 & 0 & 0 & 0 & 0 & 0 & 0 & 0 & 0 & 0 & 0 & 0 \\ 0 & 0 & 0 & 0 & 0 & 0 & 0 & 20 & 12 & 0 & 0 & 0 & 0 & 0 & 0 & 0 & 0 & 0 & 0 & 0 \\ 0 & 0 & 0 & 0 & 0 & 0 & 0 & 0 & 20 & 11 & 0 & 0 & 0 & 0 & 0 & 0 & 0 & 0 & 0 & 0 \\ 0 & 0 & 0 & 0 & 0 & 0 & 0 & 0 & 0 & 20 & 10 & 0 & 0 & 0 & 0 & 0 & 0 & 0 & 0 & 0 \\ 0 & 0 & 0 & 0 & 0 & 0 & 0 & 0 & 0 & 0 & 20 & 9 & 0 & 0 & 0 & 0 & 0 & 0 & 0 & 0 \\ 0 & 0 & 0 & 0 & 0 & 0 & 0 & 0 & 0 & 0 & 0 & 20 & 8 & 0 & 0 & 0 & 0 & 0 & 0 & 0 \\ 0 & 0 & 0 & 0 & 0 & 0 & 0 & 0 & 0 & 0 & 0 & 0 & 20 & 7 & 0 & 0 & 0 & 0 & 0 & 0 \\ 0 & 0 & 0 & 0 & 0 & 0 & 0 & 0 & 0 & 0 & 0 & 0 & 0 & 20 & 6 & 0 & 0 & 0 & 0 & 0 \\ 0 & 0 & 0 & 0 & 0 & 0 & 0 & 0 & 0 & 0 & 0 & 0 & 0 & 0 & 20 & 5 & 0 & 0 & 0 & 0 \\ 0 & 0 & 0 & 0 & 0 & 0 & 0 & 0 & 0 & 0 & 0 & 0 & 0 & 0 & 0 & 20 & 4 & 0 & 0 & 0 \\ 0 & 0 & 0 & 0 & 0 & 0 & 0 & 0 & 0 & 0 & 0 & 0 & 0 & 0 & 0 & 0 & 20 & 3 & 0 & 0 \\ 0 & 0 & 0 & 0 & 0 & 0 & 0 & 0 & 0 & 0 & 0 & 0 & 0 & 0 & 0 & 0 & 0 & 20 & 2 & 0 \\ 0 & 0 & 0 & 0 & 0 & 0 & 0 & 0 & 0 & 0 & 0 & 0 & 0 & 0 & 0 & 0 & 0 & 0 & 20 & 1\end{array}\right]$

has the following computed eigenvalues $\sigma(W)$ :

$$
\{1,2,3,4,5,6,7,8,9,10,11,12,13,14,15,16,17,18,19,20\} .
$$

For Algorithm I the spectrum $\Omega$ to be assigned is

$$
\Omega=\{1,2,3,4,5,6,7,8,9,10,21,22,23,24,25,26,27,28,29,30\}
$$

with all multiplicities equal 1 .

Then $\Omega \cap \sigma(W)=\{1,2,3,4,5,6,7,8,9,10\}$.

The entries of the computed first row of the matrix $W-c g^{T}$ are

$\begin{array}{rl}120.000051723387 & 0 \\ -247.500243369342 & 0 \\ 396.000555157266 & 0 \\ -450.450800870721 & 0 \\ 378.378800801735 & 0 \\ -236.486822771660 & 0 \\ 108.108291772582 & 0 \\ -34.459526679174 & 0 \\ 6.891906919606 & 0 \\ -0.654731295163 & 0\end{array}$


and its eigenvalues are
1. 00000000000000
21. 00000000090067
2. 00000000000000
22. 00000004690938
3. 00000000000000
23. 00000088284309
4. 00000000000000
24. 00001038739562
5. 00000000000000
24. 99999594467154
6. 00000000000000
25. 99993877257182 .
7. 00000000000000
27. 00006858100040
8. 00000000000000
28. 00015437349975
9. 00000000000000
28. 99989411123250
10. 00000000000005
29. 99998862236297

The norm of the difference vector of the eigenvalues assigned and the eigenvalues of $W-c g^{T}$ is $O\left(2 \times 10^{-4}\right)$.

For Algorithm II the spectrum $\Omega$ to be assigned is

$$
\Omega=\{1,2,3,4,5,6,7,8,9,10\}
$$

with all multiplicities equal 2.

Then $\Omega \cap \sigma(W)=\{1,2,3,4,5,6,7,8,9,10\}$.

The entries of the computed first row of the matrix $W-c g^{T}$ are

$\begin{array}{rr}-80.000000000259 & 0 \\ -202.500000001586 & 0 \\ -216.000000003493 & 0 \\ -132.300000003715 & 0 \\ -47.628000002097 & 0 \\ -9.922500000639 & 0 \\ -1.134000000102 & 0 \\ -0.063787500008 & 0 \\ -0.001417500000 & 0 \\ -0.000007087500 & 0\end{array}$

and its eigenvalues are
0. 99999999501227
6. 00000000000000
1. 00000000000000
6. 00000001350166
2. 00000000000000
6. 99999999820177
2. 00000002630856
7. 00000000000000
2. 99999995228625
8. 00000000000000
3. 00000000000000
8. 00000000031483
4. 00000000000000
9. 00000000000000
4. 00000005701010
9. 00000000000223
4. 99999995710373
9. 99999999999965
5. 00000000000000
10. 00000000000005

The norm of the difference vector of the eigenvalues assigned and the eigenvalues of $W-c g^{T}$ is $O\left(9 \times 10^{-8}\right)$. 
Example 2. The Frank matrix

$$
F=\left[\begin{array}{rrrrrrrrrrrr}
12 & 11 & 10 & 9 & 8 & 7 & 6 & 5 & 4 & 3 & 2 & 1 \\
11 & 11 & 10 & 9 & 8 & 7 & 6 & 5 & 4 & 3 & 2 & 1 \\
0 & 10 & 10 & 9 & 8 & 7 & 6 & 5 & 4 & 3 & 2 & 1 \\
0 & 0 & 9 & 9 & 8 & 7 & 6 & 5 & 4 & 3 & 2 & 1 \\
0 & 0 & 0 & 8 & 8 & 7 & 6 & 5 & 4 & 3 & 2 & 1 \\
0 & 0 & 0 & 0 & 7 & 7 & 6 & 5 & 4 & 3 & 2 & 1 \\
0 & 0 & 0 & 0 & 0 & 6 & 6 & 5 & 4 & 3 & 2 & 1 \\
0 & 0 & 0 & 0 & 0 & 0 & 5 & 5 & 4 & 3 & 2 & 1 \\
0 & 0 & 0 & 0 & 0 & 0 & 0 & 4 & 4 & 3 & 2 & 1 \\
0 & 0 & 0 & 0 & 0 & 0 & 0 & 0 & 3 & 3 & 2 & 1 \\
0 & 0 & 0 & 0 & 0 & 0 & 0 & 0 & 0 & 2 & 2 & 1 \\
0 & 0 & 0 & 0 & 0 & 0 & 0 & 0 & 0 & 0 & 1 & 1
\end{array}\right]
$$

has the following computed eigenvalues $\sigma(W)$
0.03102805830617
1. 55398870913215
0.04950743419656
3. 51185594858076
0.08122765574367
6. 96153308556712
0.14364652066476
12. $31107740086857^{\circ}$
0.28474972048519
20. 19898864587716
0.64350531900585
32. 22889150157219

For Algorithm I the spectrum $\Omega$ to be assigned written in two columns is

$$
\Omega=\left\{\begin{array}{rr}
0.03102805830617 & 7 \\
0.04950743419656 & 8 \\
0.08122765574367 & 9 \\
0.14364652066476 & 10 \\
0.28474972048519 & 11 \\
0.64350531900585 & 12
\end{array}\right\}
$$

with all multiplicities equal 1 .

$$
\text { Then } \Omega \cap \sigma(F)=\left\{\begin{array}{l}
0.03102805830617 \\
0.04950743419656 \\
0.08122765574367 \\
0.14364652066476 \\
0.28474972048519 \\
0.64350531900585
\end{array}\right\} \text {. }
$$

The entries of the computed first row of the matrix $F-c g^{T}$ are

$$
\begin{array}{rr}
-7.766335249261 & -162.577346221521 \\
-35.308642532913 & -161.397773124918 \\
-62.761983253348 & -146.348974404763 \\
-95.091914484174 & -119.095129320986 \\
-126.374891086865 & -82.976983907449 \\
-150.199014439408 & -42.265088670901
\end{array}
$$


and its eigenvalues
0.03102815917842
6. 99999999999358
0.04950736946874
8. 00000000005549
0.08122767023319
8. 99999999986791
0.14364651222413
10. 00000000014808
0.28474972064216
10. 99999999992647
0.64350531900500
11. 99999999999584

The norm of the difference vector of the eigenvalues assigned and the eigenvalues of $F-c g^{T}$ is $O\left(1.5 \times 10^{-7}\right)$.

For Algorithm II the spectrum $\Omega$ to be assigned is

$$
\Omega=\left\{\begin{array}{r}
1.55398870913215 \\
\text { 3. } 51185594858076 \\
6.96153308556712 \\
12.31107740086857 \\
\text { 20. } 19898864587716 \\
32.22889150157219
\end{array}\right\}
$$

with all multiplicities equal 2.

$$
\text { Then } \Omega \cap \sigma(F)=\left\{\begin{array}{r}
1.55398870913215 \\
3.51185594858076 \\
6.96153308556712 \\
12.31107740086857 \\
20.19898864587716 \\
32.22889150157219
\end{array}\right\} \text {. }
$$

The entries of the computed first row of the matrix $F-c g^{T}$ are

$$
\begin{array}{rr}
87.53267058319 & 2379.73451460592 \\
-255.22818519940 & -2175.29690462507 \\
656.26133592782 & 1757.99622044554 \\
-1193.96118935235 & -1255.19608884964 \\
1806.75457380974 & 809.50021222572 \\
-2234.69607175733 & -478.49127345807
\end{array}
$$

and its eigenvalues are
1. 55398866499479
1. 55398875326951
3. $51185594858072-0.00000024596755 i$
3. $51185594858072+0.00000024596755 i$
6. $96153308556700-0.00000063665662 i$
6. $96153308556700+0.00000063665662 i$
12. $31107740086755-0.00000160986180 i$.
12. $31107740086755+0.00000160986180 i$
20. 19898650094308
20. 19899079082090
32. 22888869444581
32. 22889430868959

The norm of the difference vector of the eigenvalues assigned and the eigenvalues of $F-c g^{T}$ is $O\left(5 \times 10^{-6}\right)$. 
Acknowledgments. The authors would like to thank B. N. Datta and the referees for several useful comments that improved the first version of this paper.

\section{REFERENCES}

[1] M. ARnOld AND B. N. DATTA, An algorithm for multi-input eigenvalue assignment problem, IEEE Trans. Auto. Control, 35 (1990), pp. 1149-1152.

[2] B. N. DATTA, An algorithm to assign eigenvalues in a Hessenberg matrix: Single input case, IEEE Trans Auto. Control, 5 (1987), 414-417.

[3] —, Parallel and large-scale matrix computations in control: some ideas, Linear Algebra Appl., 121 (1989), pp. 243-264.

[4] B. N. DATTA AND K. DATTA, Efficient parallel algorithms for controllability and eigenvalue assignment problems, Proc. 25th IEEE Conf. Decision and Control, Athens, Greece, 1986, pp. 1611-1616.

[5] J. KAUTSKy, N. NiChOlS, AND P. VANDOOREN, Robust pole assignment in linear feedback, Internat. J. Control, 41 (1985), pp. 1129-1155.

[6] M. Konstantinov, P. PetKov, AND N. Christov, Synthesis of linear systems with desired equivalent form, J. Assoc. Comput. Mach., 6 (1980), pp. 27-35.

[7] G. Miminis AND C. C. PAIGE, An algorithm for pole-assignment of time invariant linear system. Internat. J. Control, 35 (1981), pp. 130-138.

[8] C. Moler, J. Little, S. Bangert, And S. Kleiman, PC-MATLAB User's Guide, The MathWorks Inc., Sherborn, MA, 1986.

[9] R. V. PATEL AND P. MISRA, Numerical algorithm for eigenvalue assignment by state feedback. Proc. IEEE 72, 12 (1984), pp. 1755-1764.

[10] W. M. Wonham, Linear Multivariable Control: A Geometric Approach, Springer-Verlag, New York, 1979. 
Reproduced with permission of the copyright owner. Further reproduction prohibited without permission. 